\title{
Ex vivo Determination of an Estradiol Analogue-Induced Changes on Platelet Morphology and Angiogenic Biomarkers
}

\author{
Lisa Repsold, Etheresia Pretorius, and Annie M. Joubert ${ }^{*}$ \\ Department of Physiology, School of Medicine, Faculty of Health Sciences, University of Pretoria, Pretoria, Gauteng 0001, South Africa
}

\begin{abstract}
Angiogenesis is a closely controlled biological process that takes place during fetal development of blood vessels and wound healing, and includes the development of new blood vessels from preexisting blood vessels. Tumor angiogenesis is a means by which tumors obtain oxygen, nutrition and promote tumor growth. Angiogenesis-regulating proteins are therefore ideal biomarkers in the study of tumor pathophysiology. In our laboratory, a new in silico-designed analogue of 2-methoxyestradiol has been synthesized with angiogenic properties, namely 2-ethyl-3-O-sulfamoyl-estra-1,3,5(10)16-tetraene (ESE-16). The ex vivo influence of ESE-16 on angiogenesis and morphology in platelets of healthy participants was investigated. Scanning electron microscopy revealed no morphological changes in ESE-16-treated platelets. The possible antiangiogenic effect of ESE-16-exposed platelets was determined by means of flow cytometry measurement of angiogenic protein levels, which were significantly increased after platelets were added to tumorigenic breast epithelial cells. This indicates that binding of platelets to cancer cells causes differential release of platelet constituents. Vascular endothelial growth factor levels were decreased in platelets, whereas platelet-derived growth factor and matrix metallopeptidase- 9 levels were not significantly affected in platelets. In light of the above-mentioned data, further investigation of ESE-16's influence on morphology and angiogenic markers in platelets of cancer patients is warranted.
\end{abstract}

Key words: angiogenesis-regulating proteins, ex vivo, tumors, ESE-16, platelets

\section{INTRODUCTION}

Angiogenesis is a closely controlled biological process, which takes place during fetal development of blood vessels and wound healing (Folkman \& Shing, 1992; Yue et al., 1997). It is also an essential requirement to enable tumor growth (Yue et al., 1997; Hall et al., 2013). Tumor angiogenesis, the process leading to the formation of new blood vessels within the tumor mass, provides tumor cells with oxygen and nutrition and plays a central role in tumor cell survival (Yue et al., 1997; Ciardiello et al., 2001). It also promotes tumor growth and possible development of distant metastasis (Folkman \& Shing, 1992; Yue et al., 1997; Dunn et al., 2000; Ciardiello et al., 2001).

Platelets have been associated with malignant diseases since the late 19th century (Jain et al., 2010; Sabrkhany et al., 2011; Peterson et al., 2012). It has been hypothesized that tumors promote platelet activation and aggregation by expressing or releasing platelet-activating factors such as tissue factor, thrombin, and adenosine diphosphate (ADP) (Sabrkhany et al., 2011). Activation of platelets results in the release of platelet microparticles containing angiogenesisregulating factors, namely platelet-derived growth factor (PDGF), epidermal growth factor (EGF), matrix metallopeptidase-9 (MMP-9), and vascular endothelial growth factor (VEGF) (Wartiovaara et al., 1998; Baj-Krzyworzeka et al., 2002; Trikha et al., 2002; Jain et al., 2010; Sabrkhany et al., 2011).

Previous studies suggest that platelets not only extend the growth of tumors, but also provide tumor cells with physical and mechanical support to evade the immune system and to metastasize (Trikha et al., 2002; Jain et al., 2010). When tumor cells enter the blood stream, they undergo a variety of cell-cell interactions that are critical in providing an opportunity for tumor cells to be directly confronted with effectors of the host immune response (Nieswandt et al., 1999).

Natural killer (NK) cells provide the most effective antitumor cell activity in the blood stream and are largely responsible for the elimination of tumor cells from the circulation (Nieswandt et al., 1999; Jain et al., 2010; Coupland et al., 2013). It has been proposed that platelets form a "cloak" around tumor cells and protect the tumor cell from NK cells by providing a physical barrier and also by placing major histocompatibility complex class I antigens in the vicinity of the tumor cell surface (Nieswandt et al., 1999; Jain et al., 2010). The latter could possibly downregulate NK cytotoxic activity (Nieswandt et al., 1999; Jain et al., 2010). This was demonstrated in vitro and in vivo by Nieswandt et al. (1999). Transforming growth factor- $\beta$ (TGF- $\beta$ ), another angiogenic-regulating factor released by platelets, 
also plays a role in inhibition of the antitumor activity of T cells, NK cells, neutrophils, monocytes, and macrophages involved in regulating tumor progression (Bierie \& Moses, 2010; Pandey \& Rizvi, 2011; Pretorius, 2013).

Proteins shown to play a crucial role in the regulation of blood vessels development can be categorized into two groups such as angiogenic activators and the angiogenic inhibitors (Italiano et al., 2008; Sabrkhany et al., 2011). Angiogenesisactivating proteins include, among others, VEGF, basic fibroblast growth factor, EGF, PDGF, and MMPs (Ciardiello et al., 2001; Sierko \& Wojtukiewicz, 2004; Sabrkhany et al., 2011). Angiogenic inhibitors include endostatin, TGF- $\beta$, platelet factor-4, thrombospondin-1, and angiostatin (Sierko \& Wojtukiewicz, 2004; Sabrkhany et al., 2011).

VEGF is a dimeric glycoprotein and a member of the PDGF family that contributes to angiogenesis by promoting endothelial cell growth, maturation and survival, enhancing vascular permeability, and inhibiting apoptosis (Plake \& Warnke, 1997; Ciardiello et al., 2001; Lee et al., 2006; Hall et al., 2013). A wide variety of human tissues express low levels of VEGF. High levels are produced where angiogenesis is required such as in fetal tissue, the placenta, the corpus luteum, and in a vast majority of human tumors (Papetti \& Herman, 2002). Studies have shown that cancer patients have increased serum VEGF levels when compared with healthy individuals (Duque et al., 1999; Ciardiello et al., 2001; Peterson et al., 2012).

PDGF is found in a number of cells including platelets, fibroblasts, keratinocytes, myoblasts, astrocytes, epithelial cells, and macrophages (Papetti \& Herman, 2002). Expression of PDGF and its receptors (PDGFRs) are dynamic; their biosynthesis and processing are controlled at multiple levels (Andrae et al., 2008) and can be upregulated by a variety of stimuli including hypoxia, thrombin, cytokines, and growth factors such as TGF- $\beta$ (Andrae et al., 2008). Studies have shown increased PDGF signaling affected tumor growth, angiogenesis, invasion, and metastasis (Andrae et al., 2008). This may be explained by the fact that both PDGFR- $\alpha$ and PDGFR- $\beta$ engage in several well-characterized signaling pathways including rat sarcoma mitogen-activated protein kinase and phosphatidylinositide 3-kinase that are known to be involved in multiple cellular and developmental responses (Andrae et al., 2008).

TGF- $\beta$ is expressed by a wide variety of normal and transformed cells. TGF- $\beta$ receptors are broadly expressed in virtually all mammalian and avian cells (Papetti \& Herman, 2002). Although being characterized as an angiogenic inhibitor, it plays a vital role in angiogenesis with recent studies showing its involvement in the regulation of tumor initiation, progression, and metastasis (Bierie \& Moses, 2010; Gibbons, 2010). TGF- $\beta$ has been linked to tumor progression through the modification of carcinoma cell behavior and interaction between carcinoma cells and adjacent cell populations within the tumor microenvironment (Bierie \& Moses, 2010). TGF- $\beta$ activation can be induced by integrins, calpain, cathepsin D, kallikrein, MMP-9, plasmin, and reactive oxygen free radicals (Bierie \& Moses, 2010).
Once activated, TGF- $\beta$ binds to type II TGF- $\beta$ receptor (T $\beta$ RII). The ligand bound T $\beta$ RII is able to transactivate type I TGF- $\beta$ receptor (Bierie \& Moses, 2010). Activation of signaling occurs through serine threonine kinase activity (Bierie \& Moses, 2010).

MMPs are proteolytic enzymes that break down the extracellular matrix and growth factors/cytokines that promote tumor growth (Yoon et al., 2003). MMPs play an important role in endothelial cell proliferation and migration (Sabrkhany et al., 2011).

Angiogenesis-regulating proteins are involved in the progression of tumors (Peterson et al., 2012). These proteins are thus ideal biomarkers in studying pathophysiology of tumors by investigating the role of angiogenesis regulatory factors in platelets, lymphocytes, and erythrocytes. Therefore, the discovery of compounds that can inhibit angiogenic stimulators may play a significant role in hindering tumor growth.

In our laboratory, new in silico-designed analogues of 2-methoxyestradiol (2-ME) were synthesized (Stander et al., 2011). These agents suppress microtubule dynamics in vitro that cause mitotic spindle disruption in dividing cells and inhibits angiogenesis as it inhibits hypoxia-inducible factor$1 \alpha$ expression responsible for the secretion of VEGF, a potent angiogenic agent (Mabjeesh et al., 2003; Stander et al., 2012; Stander et al., 2013).

Scanning electron microscopy (SEM) was used to assess morphological changes in platelets after exposure to 2-ethyl-3-O-sulfamoyl-estra-1,3,5(10)16-tetraene (ESE-16) (Fig. 1). The antiangiogenic effects of ESE-16 when exposed to platelets were subsequently determined by means of flow cytometry measurement of VEGF, PDGF, and MMP-9 levels. The efficacy of this novel antitumor compound was investigated ex vivo on the platelets of three healthy individuals to determine the possible involvement of angiogenesis in platelets ex vivo after exposure to ESE-16.

\section{Materials And Methods}

Ethical approval was obtained from the Faculty of Health Sciences Research Ethics Committee with ethics reference number: 289/2013.

\section{Study Design and Population}

Blood was collected from three healthy female individuals (aged between 20 and 45 years) who did not smoke, did not use any medication, and did not suffer from chronic or acute illnesses. Participants did not have a history of autoimmune diseases, hereditary diseases, hypertension, and did not take contraceptives. Informed consent was obtained from the individuals before collection of blood and blood samples were taken after an 8-h period of fasting between 08:00 and 09:00 am. Whole blood was collected in ethylenediaminetetraacetic acid tubes and platelet-rich plasma was obtained by centrifuging the blood at $1,000 \mathrm{rpm}$ for $2 \mathrm{~min}$ and collecting plasma from separated blood. SEM was used to 

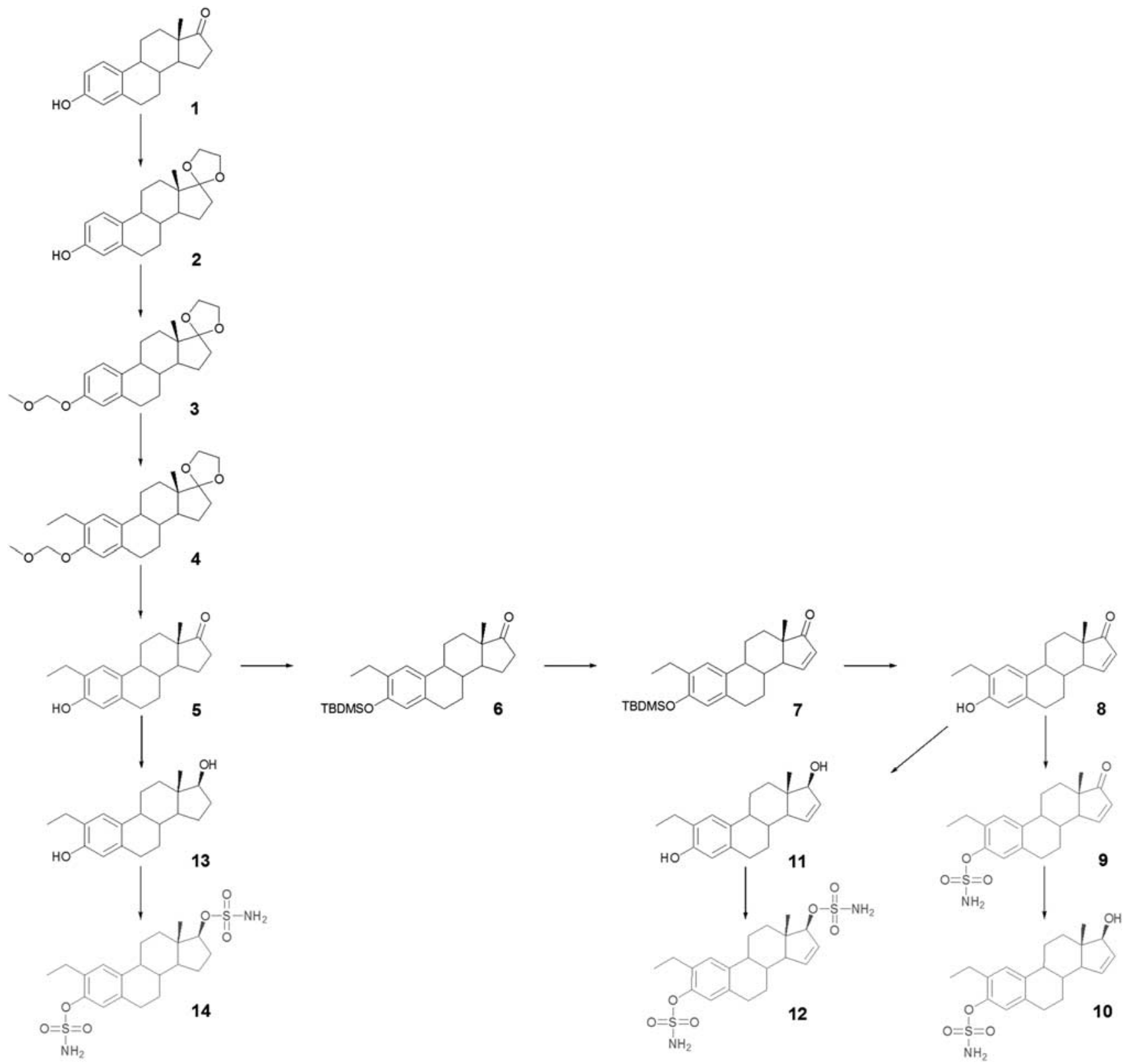

Figure 1. 2-Methoxyestradiol derived compounds indicating the structure of 2-ethyl-3-O-sulfamoyl-estra-1,3,5(10)16tetraene (step 12) (Stander et al., 2011).

assess morphological changes of platelets induced by ESE-16. The antiangiogenic effect of ESE-16, when exposed to platelets, was subsequently determined by means of flow cytometry of VEGF, PDGF, and MMP-9 levels (R\&D systems, Inc., Minneapolis, MN, USA).

\section{Preparation of Compound}

ESE-16 was in silico designed at the Bioinformatics and Computational Biology Unit, Department of Biochemistry, University of Pretoria, South Africa (Stander et al., 2011). Synthesis of ESE-16 was conducted by iThemba Pharmaceuticals (Pty) Ltd. (Modderfontein, Midrand, South Africa).

ESE-16 was dissolved in dimethyl sulfoxide (DMSO) and the final concentration did not exceed $0.01 \%(\mathrm{v} / \mathrm{v})$ in subsequent experiments. Platelets were exposed to $0.18 \mu \mathrm{M}$ of ESE-16 (Stander et al., 2011). This concentration was selected as it was previously established that $0.18 \mu \mathrm{M}$ of ESE-16 inhibited cell growth by $50 \%\left(\mathrm{GI}_{50}\right)$ after $24 \mathrm{~h}$ at $37^{\circ} \mathrm{C}$ (Stander et al., 2011; Stander et al., 2012; Stander et al., 2013). Controls included DMSO at a concentration of $0.01 \%$ as vehicle control, untreated platelets as negative control, 2-methoxyestradiol-bis-sulfamate-treated (2MEBM) platelets at a concentration of $1 \mu \mathrm{M}$, and platelets treated with $4 \%$ DMSO as positive controls (Brecher \& Cronkite, 1950; Seegers et al., 1997). As a positive control for determination of the angiogenic biomarkers VEGF, PDGF, and MMP-9, platelets were incubated with Michigan Cancer Foundation-7 (MCF-7) cells for $24 \mathrm{~h}$, whereafter exposure to the different controls followed for $24 \mathrm{~h}$. 


\section{Materials}

Phosphate-buffered saline (PBS) was purchased from GibcoBRL (Invitrogen, Carlsbad, CA, USA). DMSO was supplied by Sigma-Aldrich Co. (St. Louis, MO, USA). Angiogenic biomarkers were purchased from $R \& D$ systems Inc., Minneapolis, MN, USA. The FC500 flow cytometer equipped with a $488 \mathrm{~nm}$ air-cooled argon laser was purchased from Beckman Coulter South Africa (Pty) Ltd. (Pretoria, South Africa).

\section{Cell Line and Maintenance}

The MCF-7 cell line is an estrogen receptor-positive tumorigenic adherent breast epithelial cell line formed by pleural effusion of human breast adenocarcinoma derived from the metastatic sites in adenocarcinomas (Stander et al., 2011). MCF-7 cells were obtained from Highveld Biological (Pty) Ltd. (Sandringham, South Africa). Cells were maintained at $37^{\circ} \mathrm{C}$ in a humidified incubator with $5 \% \mathrm{CO}_{2}$. Cells were grown in $25 \mathrm{~cm}^{2}$ tissue cell culture flasks in Dulbecco's Modified Eagle Medium (DMEM) supplemented with fetal calf serum (FCS), fungizone $(250 \mu \mathrm{g} / \mathrm{L})$, streptomycin $(100 \mu \mathrm{g} / \mathrm{mL})$, and penicillin $\mathrm{G}(100 \mathrm{U} / \mathrm{mL})$. Upon complete colonization of the cell culture flasks, cells were dispersed by trypsinization, which entailed incubation with trypsin for 2-5 min, removal thereof, and resuspension with DMEM into separate subcultures for further culturing or use in experiments.

For experimentation, cells were seeded at 500,000 cells/ $25 \mathrm{~cm}^{2}$ cell culture flask, left to attach for $24 \mathrm{~h}$ and subsequent exposure with $1,5 \times 10^{8}$ platelets $/ \mathrm{mL}$ to ESE-16, $0.1 \%$ DMSO as vehicle control, cells and platelets exposed to growth medium only, and cells and platelets exposed to $4 \% \mathrm{DMSO}$ as a positive control for $24 \mathrm{~h}$ (Radziwon-Balicka et al., 2012; Radziwon-Balicka et al., 2013). Before exposure, cells were washed thoroughly with PBS to remove all serum contaminants and subsequently DMEM without FCS was added, followed by platelets. FCS was excluded from the platelet studies as it is known to contain growth factors (including VEGF, PDGF, and TGF). Therefore, FCS was not included to ensure results originate from tumor cell-platelet interaction and not from traces of serum from supplemented DMEM (Childs et al., 1982; Grabowski et al., 1996; Mannello \& Tonti, 2007; Oida \& Weiner, 2010; van der Valk et al., 2010).

\section{SEM}

Morphology of platelet samples exposed to ESE-16 was viewed with a Zeiss ULTRA plus FEG-SEM at the Microscopy and Microanalysis Unit of the University of Pretoria, Pretoria, South Africa and the National Centre for NanoStructured Materials of the Council for Scientific and Industrial Research.

Ex vivo samples were prepared on glass plates with $10 \mu \mathrm{L}$ platelets as a control, $10 \mu \mathrm{L}$ platelets exposed to ESE-16, $10 \mu \mathrm{L}$ platelets exposed to DMSO, and $10 \mu$ l platelets exposed to positive control 2MEBM and 4\% DMSO (Pretorius, 2013). Glass plates with ex vivo samples were placed in six-well plates and left to dry slightly, after which the samples were washed for $20 \mathrm{~min}$ in 50\% PBS:50\% distilled $\mathrm{H}_{2} \mathrm{O}$ solution. Samples were fixed with glutaraldehyde and PBS for $30 \mathrm{~min}$ and washed three times in PBS for 3 min each for subsequent secondary fixation in osmium for $15 \mathrm{~min}$. Samples were washed three times each for $3 \mathrm{~min}$ and dehydrated for $3 \mathrm{~min}$ each in increasing concentrations of ethanol, $30 \%, 50 \%, 70 \%$, $90 \%$, and three times in 100\% ethanol (Pretorius, 2013). Samples were critical point dried, mounted, carbon coated, and viewed with the Zeiss ULTRA plus FEG-SEM (Carl Zeiss (Pty) Ltd., Johannesburg, South Africa).

\section{Flow Cytometry Measurement of Angiogenic Biomarkers}

\section{VEGF Measurement}

Platelets are the major physiological transporter of VEGF in blood (Wartiovaara et al., 1998; Sabrkhany et al., 2011; Coupland et al., 2013). This angiogenic factor is released from platelet granules following activation of platelets (Wartiovaara et al., 1998). The experiment was designed to identify and quantify blood cells (via flow cytometry) expressing cytoplasmic forms of the VEGF protein, which is recognized by the antihuman VEGF-allophycocyanin monoclonal antibody.

Platelets were collected in evacuated citrate tubes and exposed to ESE-16, and controls (described above) for the appropriate time period. Samples were washed thrice using PBS containing $0.5 \%$ bovine serum albumin (BSA) and centrifuged for $5 \mathrm{~min}$ at $500 \times g$. Packed samples $(50 \mu \mathrm{L})$ were transferred to a new $5 \mathrm{~mL}$ tube. Samples were washed with PBS and centrifuged for $7 \mathrm{~min}$ at $200 \times g$. Samples $(500,000)$ were resuspended in $0.5 \mathrm{~mL}$ cold $4 \%$ paraformaldehyde and incubated for $10 \mathrm{~min}$ at room temperature. Samples were washed with PBS and centrifuged for $7 \mathrm{~min}$ at $200 \times g$. Supernatant was discarded and the pellet was resuspended in $2 \mathrm{~mL}$ saponin (SAP) buffer (Hanks' Balanced Salt Solution containing $0.1 \%$ saponin, $0.05 \% \mathrm{NaN}_{3}$ ). Samples were centrifuged for $7 \mathrm{~min}$ at $200 \times g$ and the supernatant was discarded, whereas ensuring that $200 \mu \mathrm{L}$ SAP buffer remained in the tube. Samples were resuspended with $10 \mu \mathrm{L}$ allophycocyanin-conjugated antihuman VEGF by briefly vortexing and were then incubated for $30-45 \mathrm{~min}$ in the dark. Samples were washed with $2 \mathrm{~mL}$ SAP buffer, centrifuged for $7 \mathrm{~min}$ at $200 \times \mathrm{g}$, resuspended in $200-400 \mu \mathrm{L}$ PBS and analyzed with the FC500. Information generated from at least 10,000 events was analyzed by means of Cyflogic version 1.2.1 software (Pertu Therho, Turko, Finland).

\section{PDGF- $\alpha$ and $-\beta$}

Expression of PDGFR- $\alpha$ has been noticed in subtypes of mesenchymal progenitors in the lungs, skin, and intestine (Peterson et al., 2012). PDGFR- $\beta$ is expressed in mesenchyme, particularly in vascular SMCs and pericytes (Andrae et al., 2008). 
According to R \& D Systems, this protocol can be used to quantitatively determine the percentage of cells bearing the PDGFR- $\alpha$ and $-\beta$ and to quantitatively determine the density of these receptors on the cell surface via flow cytometry. This was conducted with the use of a fluoresceinlabeled antibody, which binds to the cells expressing the receptors. The fluorescence intensity is directly proportional to the density of expression of the receptors on the cells and was determined by monitoring the fluorescence emitted after $488 \mathrm{~nm}$ wavelength laser excitation.

\section{PDGFR- $\alpha$}

Platelet samples were centrifuged for $5 \mathrm{~min}$ at $500 \times \mathrm{g}$ and samples were washed three times with isotonic phosphate buffer containing $0.5 \%$ BSA. Samples were treated with $1 \mu \mathrm{g}$ of human IgG/ $10^{5}$ cells for $15 \mathrm{~min}$ at room temperature to $\mathrm{Fc}$ block the samples. The sample ( $50 \mu \mathrm{l})$ was then transferred to a $5 \mathrm{~mL}$ tube for staining. Carboxyfluorescein (CFS)conjugated PDGFR- $\alpha(10 \mu \mathrm{l})$ was added to samples after an incubation period of $30-45 \mathrm{~min}$ at $2-8^{\circ} \mathrm{C}$, samples were washed twice with $4 \mathrm{~mL}$ PBS to remove unattached antibodies (De Boeck \& Dijke, 2012; Guijarro-Muñoz et al., 2012). Samples were resuspended in $200-400 \mu \mathrm{L}$ PBS and analyzed with the FC500. Information generated from at least 10,000 events was analyzed by means of Cyflogic version 1.2.1 software.

\section{PDGFR- $\beta$}

Platelet samples were centrifuged for $5 \mathrm{~min}$ at $500 \times g$ and washed three times with isotonic phosphate buffer containing $0.5 \%$ BSA. A minimum of a 1,000,000 platelets was transferred to a $5 \mathrm{~mL}$ tube for staining. CFS-conjugated PDGFR- $\beta(10 \mu \mathrm{L})$ was added to the samples after an incubation period of $30 \mathrm{~min}$ at room temperature and the samples were washed twice with $4 \mathrm{~mL}$ PBS to remove unattached antibodies (De Boeck \& Dijke, 2012; Guijarro-Muñoz et al., 2012). Samples were resuspended in flow cytometry staining buffer and analyzed with the FC500 as described above.

\section{MMP-9}

MMP-9 is another angiogenic-regulating factor transported by platelets (Yoon et al., 2003). This experiment was designed to identify and quantitate cells expressing MMP-9 with the binding of an antihuman MMP-9-fluorescein monoclonal antibody to the MMP-9 protein (Bruserud et al., 2010; Reikvam et al., 2010).

Platelets were collected in evacuated tubes containing citrate. In order to remove contaminating serum components, samples were washed twice in PBS and then centrifuged at $200 \times g$ for $7 \mathrm{~min}$. Subsequently, 500,000 platelets was fixed in cold paraformaldehyde (4\%) and incubated at room temperature for $10 \mathrm{~min}$. Platelets were vortexed, washed twice in PBS, and centrifuged at $200 \times g$ for $7 \mathrm{~min}$. The platelet pellet was then resuspended in $2 \mathrm{~mL} \mathrm{SAP}$ buffer and centrifuged at $200 \times g$ for $7 \mathrm{~min}$.
After centrifuging, the supernatant was discarded with $200 \mu \mathrm{L}$ of the SAP buffer remaining in the tube. Platelets in the $200 \mu \mathrm{L}$ SAP buffer were resuspended and $10 \mu \mathrm{L}$ of the antibody conjugate was added (Bruserud et al., 2010; Reikvam et al., 2010). Samples were vortexed and incubated for 30-45 min in the dark at room temperature. After incubation, samples were washed twice with $2 \mathrm{~mL}$ of SAP buffer and centrifuged at $200 \times g$ for $7 \mathrm{~min}$. The supernatant was discarded, samples were resuspended in $200 \mu \mathrm{l}$ of PBS and samples were analyzed with the FC500 as described above.

\section{Statistics}

Quantitative and qualitative data were obtained. Qualitative data included SEM images that were confirmed by quantitative data. Quantitative data included flow cytometry measurement of angiogenic biomarkers VEGF, PDGF, and MMP-9. Samples consisted of platelets collected from three healthy individuals and data were expressed as a ratio of the value measured for the ESE-16-treated samples compared with the vehicle-treated exposed samples defined as mean relative fluorescence. This involved flow cytometry analysis of at least 10,000-30,000 events that was repeated in triplicate (three independent experiments), whereafter a representative figure was chosen for each experiment with the use of Cyflogic version 1.2.1 software, which calculates the means of $X$ and $Y$ co-ordinates for each treatment. Statistical analysis consisted of ANOVA Student's $t$-test of which a $p$-value of $<0.05$ was considered to be statistically significant to compare the assessment results for the treatment groups and biological variations. Standard deviations were also determined as indicated in the bar graphs. Statistically insignificant data obtained are of utmost importance to prove the hypothesis that ESE-16 does not cause damage to platelets per se.

\section{Results}

\section{SEM}

With the use of SEM, the surface structure and morphology of biological structures can be viewed to determine potential morphological changes (Pretorius, 2011). SEM provides highquality, high-resolution images of platelets exposed to ESE-16 and appropriate controls. These images are shown in Figure 2 as representative of the three participants and indicate that platelets are not activated after exposure to ESE-16. Activation of platelets is clearly shown in the positive control images (Figs. $2 \mathrm{~d}, 2 \mathrm{e}$ ), with spreading and formation of fibrin networks, indicating initiation of coagulation.

\section{VEGF}

Isolated platelets were exposed to ESE-16 and VEGF expression was measured with flow cytometry. Results (Fig. 3) indicated a slight decrease in VEGF levels in ESE-16-treated platelets when compared with the vehicle control samples. 

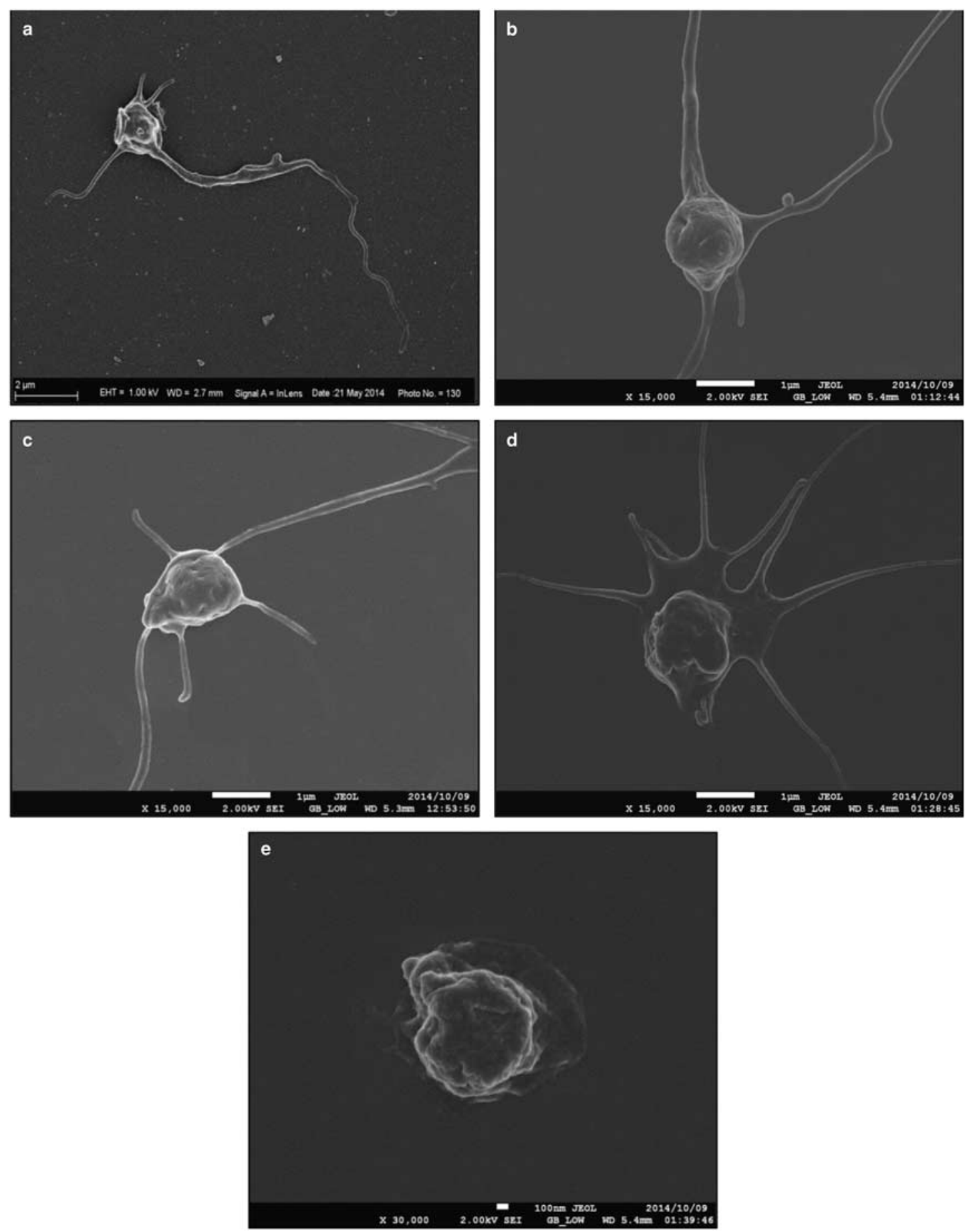

Figure 2. Scanning electron microscopy images of platelets exposed to 2-ethyl-3-O-sulfamoyl-estra-1,3,5(10)16tetraene (ESE-16) and various controls. a: Control platelet sample indicating normal morphology of platelets. b: Dimethyl sulfoxide (DMSO)-exposed samples. c: ESE-16-treated platelets indicating that the morphology of platelets was not affected following exposure to ESE-16. d: 2-Methoxyestradiol-bis-sulfamate-treated platelets. e: Platelets treated with $4 \%$ DMSO as a positive control for platelet damage.

To substantiate these results and confirm the expression of VEGF, platelets were incubated with MCF-7 cells for $24 \mathrm{~h}$ and exposed as previously discussed. This caused a marked increase in VEGF levels in MCF-7 cells when platelets were added as seen in Figure 3. MCF-7 cells propagated in medium only (without platelets) revealed a mean fluorescence intensity (MFI) of 4.14 for patient 1, 5.76 for patient 2, and 5.15 for patient 3, whereas the MFI of MCF-7 cells with the addition of platelets was found to be 8.83 for patient 1, 9.06 for patient 2, and 6.7 for patient 3. Thus, expression of VEGF doubled when platelets were added to MCF-7 cells. 

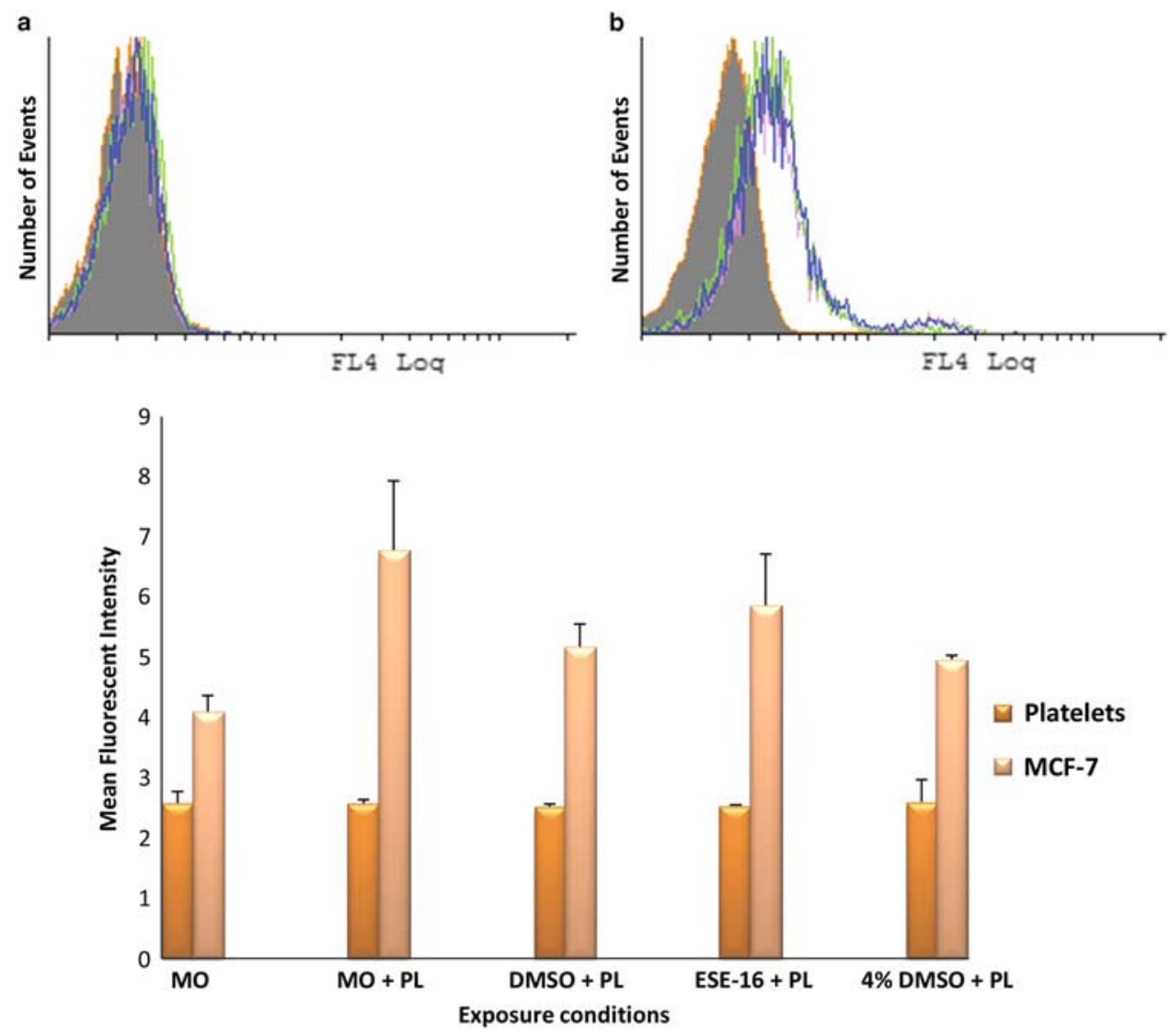

Figure 3. Overlay histograms and bar graph of vascular endothelial growth factor (VEGF) levels in platelets and MCF-7 cells exposed to 2-ethyl-3-O-sulfamoyl-estra-1,3,5(10)16-tetraene (ESE-16) and various other controls. Platelets (histogram A) and MCF-7 cells (histogram B) propagated in medium only (MO) are indicated in the orange histograms, MCF-7 cells with the addition of platelets (MO + PL) are shown by the pink histogram, the vehicle control is indicated by the green histogram, and ESE-16-treated platelets are indicated in the blue histogram. Results indicate that there was a significant increase in the MFI of VEGF in MCF-7 cells when platelets were added to MCF-7 cells (as seen when $\mathrm{MO}$ and $\mathrm{MO}+\mathrm{PL}$ results were compared). However, there was not a statistically significant increase of VEGF levels between the vehicle control platelets and ESE-16-treated platelets ( $p$-value of 0.758 in platelets and 0.270 in MCF-7 cells). Dimethyl sulfoxide (DMSO).

\section{PDGF- $\alpha$ and $-\beta$}

The expression of PDGFR- $\alpha$ and PDGF- $\beta$ in platelets was determined in ESE-16-treated platelets. Expression of PDGFR- $\alpha$ in platelets incubated with MCF-7 cells is shown in Figure 4, as an overview of all three participants, whereas PDGF- $\beta$ levels in platelets with MCF-7 cells are shown in Figure 5 for an overview of all three participants, which illustrated an increase in PDGFR- $\alpha$ and PDGF- $\beta$ levels in MCF-7 cells when platelets were added. The expression of PDGFR- $\alpha$ and PDGF- $\beta$ when platelets were added to MCF-7 cells was distinctly increased when compared with MCF-7 cells in medium only. The levels of PDGF- $\beta$ were slightly decreased in ESE-16-exposed samples when compared with the vehicle control samples (Fig. 5).

\section{MMP-9}

Platelets expressing MMP-9 were measured with the binding of an antihuman MMP-9-fluorescein monoclonal antibody to the MMP-9 protein. Results for platelets incubated with MCF-7 cells are presented in Figure 6 for a summary of all three participants. Levels of MMP-9 expressed by platelets only and that of platelets and MCF-7 cells combined were not significantly increased when compared with the vehicle control samples, but there was a slight increase in MMP-9 levels in MCF-7 cells combined with platelets when compared with MCF-7 cells propagated in medium only.

\section{Discussion}

The in silico-designed analogue of 2-ME, namely ESE-16, binds to carbonic anhydrase II (CAII), delaying early metabolism, and is thus carried in the circulation (Stander et al., 2012). The effect it potentiates on blood components, especially on platelets, is of significance in cancer progression. This study investigated the effect of the novel in silicodesigned antitumor compound ESE-16 on platelets of three 

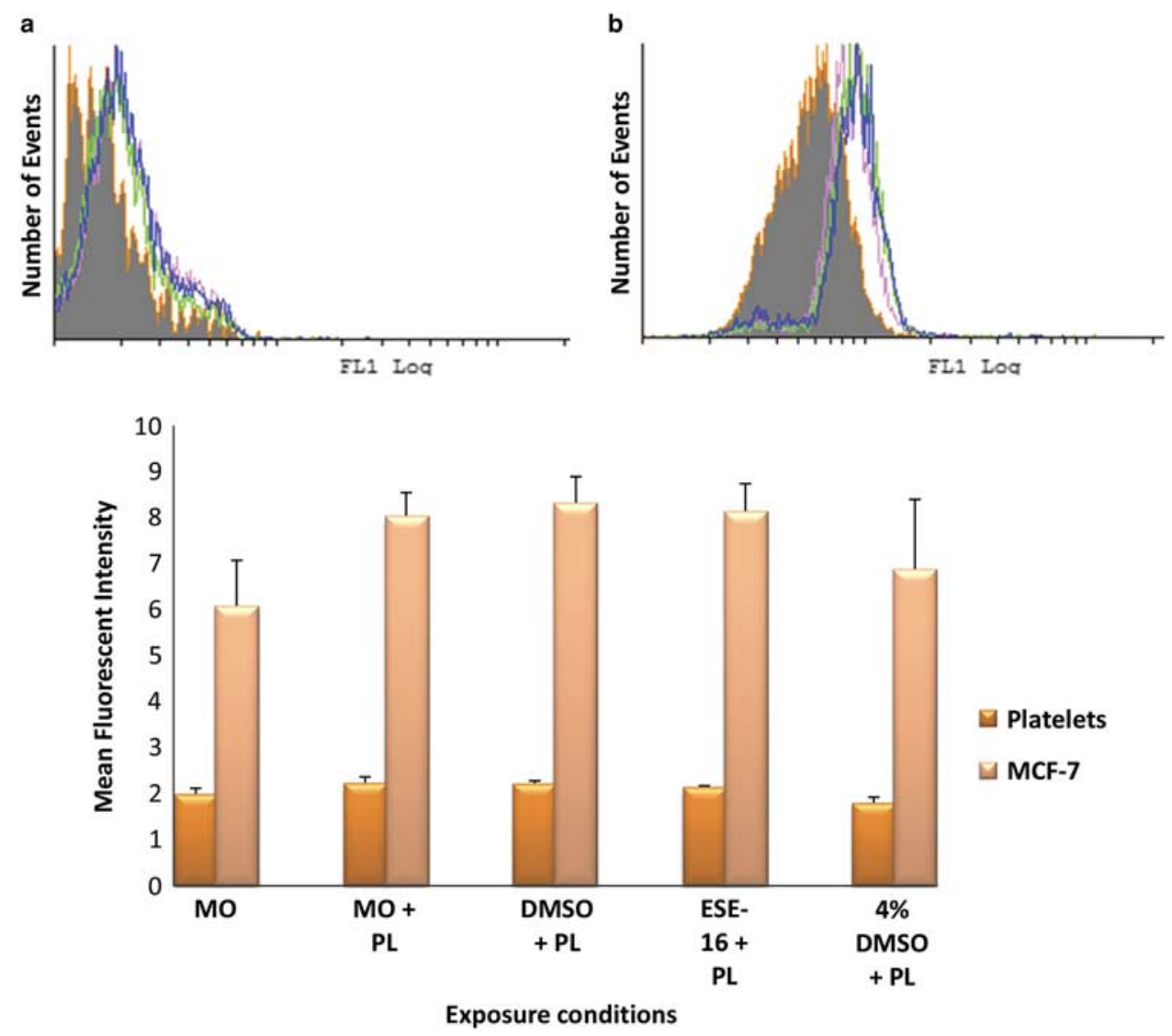

Figure 4. Overlay histograms and bar graph indicating the MFI of platelet-derived growth factor- $\alpha$ (PDGF- $\alpha$ ) levels in 2-ethyl-3-O-sulfamoyl-estra-1,3,5(10)16-tetraene (ESE-16)-treated platelets when combined with MCF-7 cells. Platelets (histogram A) and MCF-7 cells (histogram B) propagated in medium only (MO) are indicated in the orange histograms, MCF-7 cells with the addition of platelets $(\mathrm{MO}+\mathrm{PL})$ are shown by the pink histogram, the vehicle control is indicated by the green histogram, and ESE-16-treated platelets are indicated in the blue histogram. Results from the bar graph indicate that there was again a significant increase in the MFI/expression of PDGF- $\alpha$ in MCF-7 cells when platelets were added to MCF-7 cells (as seen when MO and MO + PL results were compared). There was not a statistically significant difference in PDGF- $\alpha$ levels between the vehicle control platelets and ESE-16-treated platelets ( $p$-value of 0.116 in platelets and 0.727 in MCF-7 cells). Dimethyl sulfoxide (DMSO).

healthy individuals in order to determine potential morphological changes and the influence on angiogenic biomarkers after exposure.

The antitumor abilities of this unique in silico-designed compound have been studied in vitro on various cancer cell lines in our laboratory (Stander et al., 2011; Stander et al., 2012; Stander et al., 2013). Cell lines included the tumorigenic human epithelial cervical HeLa cell line, MCF-7 breast cancer cell line, esophageal carcinoma SNO cell line, and the metastatic MDA-MB-231 breast cancer cell line (Stander et al., 2011; Stander et al., 2012; Nkandue et al., 2013; Stander et al., 2013; Theron et al., 2013). It was confirmed that the compound had similar mechanisms to that of its source compound, 2-ME, including tubulin disruption, inhibition of CAIX, and induction of apoptosis and autophagy (Stander et al., 2011; Stander et al., 2012; Nkandue et al., 2013; Stander et al., 2013; Theron et al., 2013). In addition, it was found that the compound was more potent than 2-ME with antiproliferative activity at nanomolar values, whereas $2-\mathrm{ME}$ needed a concentration between $1-2 \mu \mathrm{M}$ to have any antiproliferative effects (Stander et al., 2011).

Data from in vitro studies supported the hypothesis that this compound may act as a potential antitumor drug with antiproliferative and pro-apoptotic characteristics (Stander et al., 2011; Stander et al., 2012; Nkandue et al., 2013; Stander et al., 2013; Theron et al., 2013). Research showed that ESE-16 was an antimitotic compound and induced apoptosis in these cancer cell lines (Stander et al., 2011; Stander et al., 2012; Nkandue et al., 2013; Stander et al., 2013; Theron et al., 2013). It is important to note that ESE-16 has also been tested on nontumorigenic MCF-12 A breast cells and has shown a higher affinity for cancerous cell lines when compared with this noncancerous cell line (Stander et al., 2012; Stander et al., 2013).

Promising results obtained from the in vitro studies in our laboratory led to ex vivo studies where the possible antiangiogenic properties of ESE-16 were determined. As angiogenesis is required at almost every step of tumor progression and metastasis, tumor vasculature has been 

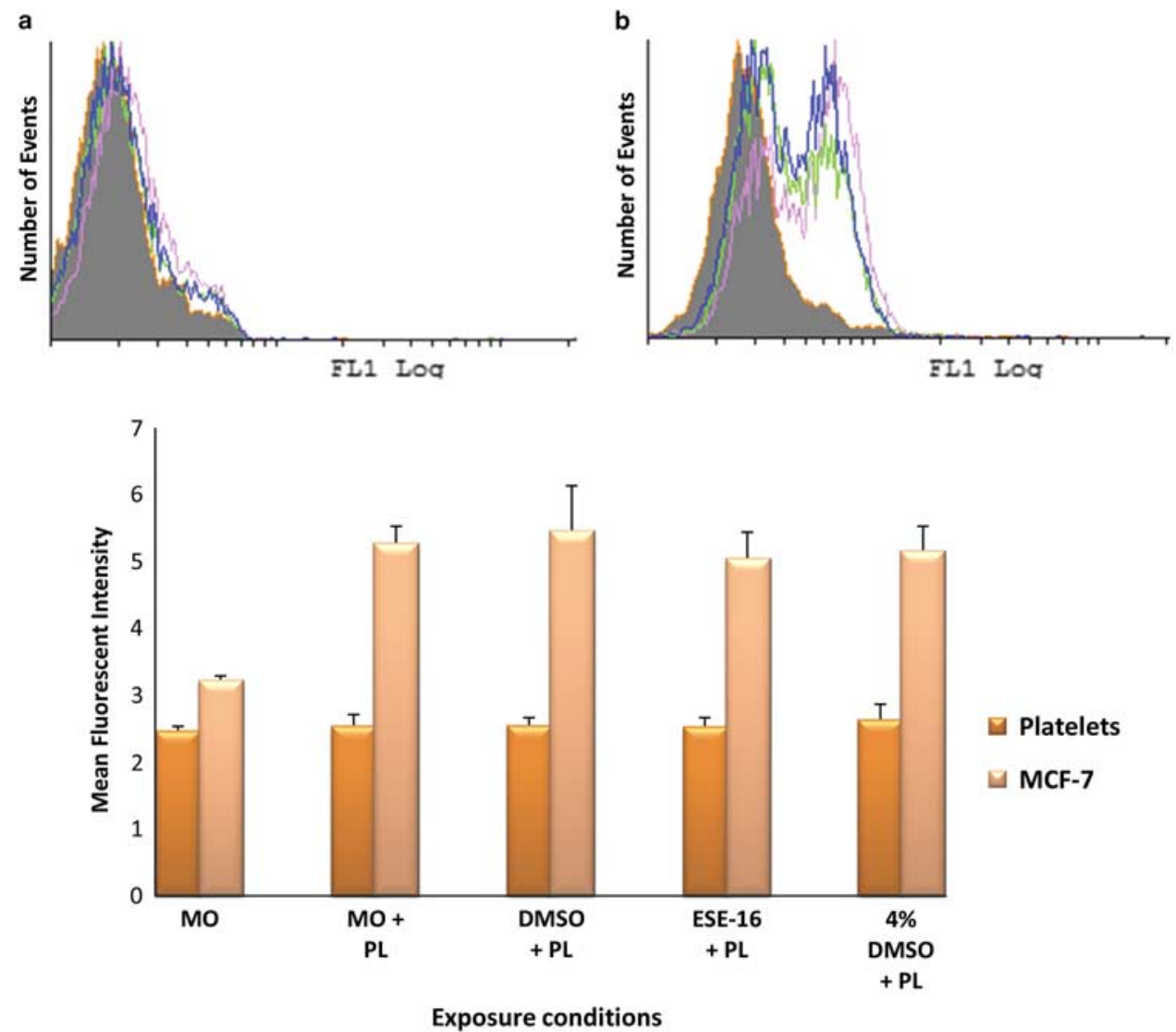

Figure 5. Overlay histograms and bar graph indicating the MFI of platelet-derived growth factor- $\beta$ (PDGF- $\beta$ ) levels in 2-ethyl-3-O-sulfamoyl-estra-1,3,5(10)16-tetraene (ESE-16)-treated platelets when combined with MCF-7 cells. Platelets (histogram A) and MCF-7 cells (histogram B) propagated in medium only (MO) are indicated by the orange histograms, MCF-7 cells with the addition of platelets (MO + PL) are shown by the pink histogram, the vehicle control is indicated by the green histogram, and ESE-16-treated platelets are indicated in the blue histogram. Results from the bar graph indicate that there was a significant increase in the MFI/expression of PDGF- $\beta$ in MCF-7 cells when platelets were added to MCF-7 cells (as seen when MO and MO + PL results were compared). A slight decrease was observed in PDGF- $\beta$ levels in ESE-16-treated platelets when compared with the vehicle control platelets ( $p$-value of 0.868 in platelets and 0.410 in MCF-7 cells). Dimethyl sulfoxide (DMSO).

identified as a strong prognostic marker for tumor grading. Inhibition of angiogenesis induced by tumors is a promising therapeutic strategy. Several antiangiogenic strategies have been developed to inhibit tumor growth by targeting different components of tumor angiogenesis (Bhat \& Singh, 2008; Pratheeshkumar et al., 2012).

With the use of SEM, possible morphological changes were studied on the platelets of healthy female individuals after exposure to ESE-16. Results showed no morphological changes in the platelets after exposure when compared with the relevant controls (Repsold et al., 2014a, 2014b).

The antiangiogenic effect of ESE-16 when exposed to whole blood was subsequently determined by means of flow cytometry measurement of levels of VEGF, PDGF, and MMP-9. As no positive control for angiogenesis induction is available for ex vivo studies, platelets were co-cultured with MCF-7 cells and expression of these angiogenic biomarkers subsequent to exposure was determined. No statistically significant difference in the ESE-16-exposed platelets was observed. However, when platelets were combined with MCF-7 cells (positive control), a statistically significant increase in the expression of these biomarkers was noted when compared with MCF-7 cells propagated in medium only.

In the case of VEGF expression, the MFI was decreased in ESE-16-treated platelets, which signify the ability of ESE-16 to function in an antiangiogenic manner. This was also the case for PDGF- $\beta$ levels, where the MFI was found to be decreased in platelets and MCF-7 cells when compared with the respective vehicle controls. PDGF- $\alpha$ and MMP-9 expression was found to be consistent between different platelet exposure conditions. However, their levels were also increased significantly when platelets were added to MCF-7 cells (positive control).

The activation of platelets by tumors takes place through direct interaction or through the release of various molecules including ADP, thrombin, MMP-2, and thromboxane A2 (Carmeliet \& Jain, 2000; Battinelli et al., 2011). Release of these mediators during platelet activation can occur into the peritumoral space, which directly enhances extravasation 

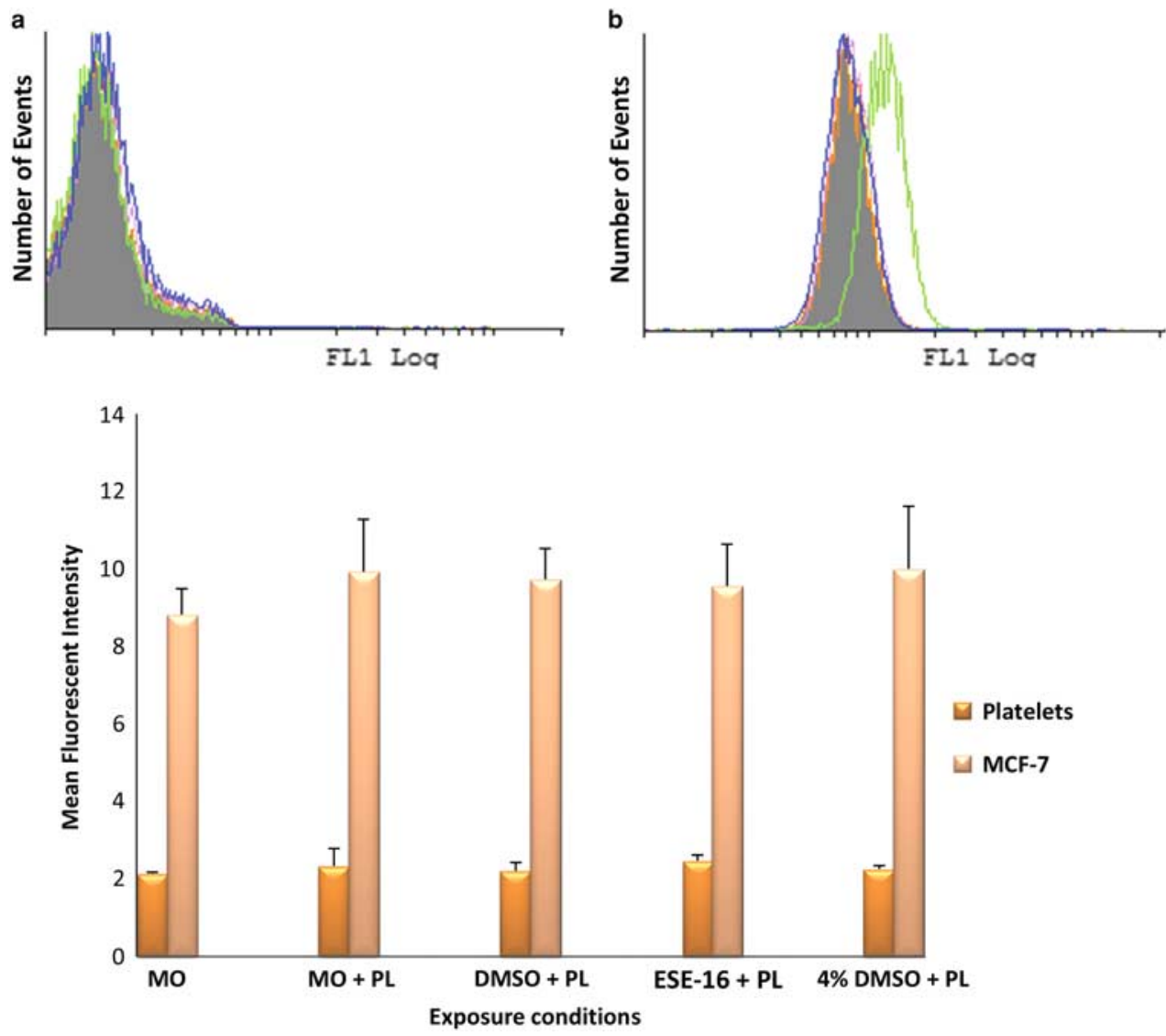

Figure 6. Overlay histograms and bar graph indicating the MFI of matrix metallopeptidase-9 (MMP-9) levels in 2-ethyl-3-O-sulfamoyl-estra-1,3,5(10)16-tetraene (ESE-16)-treated platelets when combined with MCF-7 cells. Platelets (histogram A) and MCF-7 cells (histogram B) propagated in medium only (MO) are indicated by the orange histograms, MCF-7 cells with the addition of platelets (MO + PL) are shown by the pink histogram, the vehicle control is indicated by the green histogram, and ESE-16-treated platelets are indicated in the blue histogram. Results indicate that there was a significant increase in the MFI/expression of MMP-9 in MCF-7 cells in comparison with the expression of MMP-9 by platelets. Results show that there was a statistically insignificant difference between the vehicle control platelets and ESE-16-treated platelets between all three participants. However, an increase was observed in the MFI of MMP-9 expression by MCF-7 cells when platelets were added ( $p$-value of 0.156 in platelets and 0.842 in MCF-7 cells). Dimethyl sulfoxide (DMSO).

through degradation of structural parts present in the basement membrane (Carmeliet \& Jain, 2000). This is, for example, the case in breast and ovarian cancers where exposure of these tumors to platelets increases invasiveness (Min Soon et al., 2012).

Platelet activation by tumor cells is followed by platelet aggregation during which the invasive phenotype of tumors is enhanced by increasing the expression of MMP-9 and TGF- $\beta$ (Mannello \& Medda, 2011; Yan et al., 2014). Amplification of tumor growth thus occurs by binding of tumor cells to platelets and subsequent activation of platelets. Cancer cells activate and bind to activated platelets via P-selectin expressed on platelet membranes. This results in the release of mitogens including VEGF, PDGF, and TGF- $\beta$, which increase growth and vascularization of the tumor mass. This, in turn, further activates platelets following the release of microparticles, amplifying what is known as the platelet-cancer loop. Thus, when co-incubating MCF-7 cells with platelets, the platelet-cancer loop is activated through interaction of platelets with the MCF-7 cells, thereby increasing the expression of mediators of angiogenesis from platelets.

Metastatic spread is the main source of deaths related to cancer and therefore provides an important research focus in the treatment of cancer. The role of platelets in cancer is crucial as they support growth and metastasis of tumors through the release of angiogenic proteins. Inhibition of angiogenesis induced by tumors is a promising therapeutic strategy for cancer. Several antiangiogenic strategies have been developed to inhibit tumor growth by targeting different components of tumor angiogenesis (Bhat \& Singh, 2008; Pratheeshkumar et al., 2012).

These results are in accordance with findings by Stander et al. (2012) who suggested that sulfamoylated analogues have potential antiCAIX) activity. CAIX contributes to the acidic extracellular environment associated with tumors, which in turn promotes the expression of proteinases that 
contribute to invasion and metastasis. This compound also reversibly binds to CAII in red blood cells in order to bypass the first-pass metabolism in the liver owing to release into the blood stream from CAII, resulting in increased bioavailability (Stander et al., 2012). This could explain why the compounds do not affect platelets.

This ex vivo study is the first to investigate the unique in silico-designed ESE-16 morphological and antiangiogenic effects on platelets. Results provide a point of reference for future investigations and as the compound does not cause morphological damage ex vivo and ESE-16 decreased the expression of angiogenic markers including VEGF, it warrants further in vivo studies.

\section{CONCLUSION}

This study reports that the promising $17 \beta$-estradiol analogue, ESE-16, did not cause morphological damage to platelets when exposed for $24 \mathrm{~h}$ at $0.18 \mu \mathrm{M}$. VEGF levels are expected to be higher in patients with cancer as it promotes angiogenesis. The current study revealed the levels of VEGF when exposed to ESE-16 was slightly decreased and the addition of platelets to MCF-7 cancer cells significantly increases the expression of angiogenic biomarkers VEGF, PDGF, and MMP-9. Data from this study reveals the potential of this compound to be applied to ex vivo study techniques to validate prospective in vivo studies and the chemotherapeutic potential of this possible antitumor agent in vivo.

\section{ACKNOWLEDGMENTS}

Funding was obtained by Professor A.M. Joubert from the Struwig-Germeshuysen Research Trust of South Africa, the National Research Foundation, the Cancer Association of South Africa, and the South African Medical Research Council. Preparation of blood samples was conducted at the cell culture laboratory of the Department of Physiology at the University of Pretoria, Pretoria, South Africa. Preparation of all blood samples for the scanning electron microscopy, as well as image capturing was done at the Microscopy and Microanalysis Unit of the University of Pretoria, Pretoria, South Africa, and the National Centre for Nano-structured Materials of the Council for Scientific and Industrial Research. Flow cytometry analysis was done at the Department of Pharmacology, University of Pretoria, Pretoria, South Africa.

\section{REFERENCES}

Andrae, J., Gallini, R. \& Betsholtz, C. (2008). Role of plateletderived growth factors in physiology and medicine. Genes Dev 22(10), 1276-1312.

Baj-Krzyworzeka, M., Majka, M., Pratico, D., Ratajczak, J., Vilaire, G., Kijowski, J., Reca, R., Janowska-Wieczorek, A. \& RATAJCZAK, M.Z. (2002). Platelet-derived microparticles stimulate proliferation, survival, adhesion, and chemotaxis of hematopoietic cells. Exp Hematol 30(5), 450-459.
Battinelli, E.M., Markens, B.A. \& Italiano, J.E. Jr. (2011). Release of angiogenesis regulatory proteins from platelet alpha granules: Modulation of physiologic and pathologic angiogenesis. Blood 118(5), 1359-1369.

BHAT, T.A. \& SingH, R.P. (2008). Tumor angiogenesis-A potential target in cancer chemoprevention. Food Chem Toxicol 46, 1334-1345.

Bierie, B. \& Moses, H.L. (2010). Transforming growth factor beta (TGF- $ß)$ and inflammation in cancer. Cytokine Growth Factor Rev 21, 49-59.

Brecher, G. \& Cronkite, E.P. (1950). Morphology and enumeration of human blood platelets. J Appl Physiol 3(6), 365-377.

Bruserud, O., Hatfield, K.J., Kalland, K.H., Kittang, A.O., Oyan, A.M. \& Reikvam, H. (2010). Primary human acute myelogenous leukemia cells release matrix metalloproteases and their inhibitors: Release profile and pharmacological modulation. Eur J Haematol 84, 239-251.

CARMeliet, P. \& JaIN, R.K. (2000). Angiogenesis in cancer and other diseases. Nature 407(6801), 249-257.

Childs, C.B., Proper, J.A., Tucker, R.F. \& Moses, H.L. (1982). Serum contains a platelet-derived transforming growth factor. Proc Natl Acad Sci U S A 79(17), 5312-5316.

Ciardiello, F., Caputo, R., Bianco, R., Damiano, V., Fontanini, G., Cuccato, S., De Placido, S., Bianco, A.R. \& Tortora, G. (2001). Inhibition of growth factor production and angiogenesis in human cancer cells by ZD1839 (Iressa), a selective epidermal growth factor receptor tyrosine kinase inhibitor. Clin Cancer Res 7(5), 1459-1465.

Coupland, L.A., Chong, B.H. \& Parish, C.R. (2013). Beware of NK cells in pre-clinical metastasis models. Clin Exp Metastasis 30(7), 945-947.

De Boeck, M. \& Dijke, P.T. (2012). Key role for ubiquitin protein modification TGFß signal transduction. Ups J Med Sci 117, 153-165.

DunN, I.F., HeEsE, O. \& Black, P.M. (2000). Growth factors in glioma angiogenesis: FGFs, PDGF, EGF, and TGFs. J Neurooncol 50(1-2), 121-137.

Duque, J.L., Loughlin, K.R., Adam, R.M., Kantoff, P.W., Zurakowski, D. \& Freeman, M.R. (1999). Plasma levels of vascular endothelial growth factor are increased in patients with metastatic prostate cancer. Urology 54(3), 523-527.

Folkman, J. \& SHING, Y. (1992). Angiogenesis. J Biol Chem 267(16), 10931-10934.

GibBons, J. (2010). Angiogenesis: Emerging roles for the TGF $\beta$ superfamily. Pathw Rev 11, 14-16.

Grabowski, E.F., Boor, S.E., Rodino, L.J., Jang, I.K., Gold, H. \& Michelson, A.D. (1996). Platelets are degranulated by some, but not all, contrast media. Acad Radiol 3(2), S328-S330.

Guijarro-Muñoz, I., Cuesta, A.M., Alvarez-Cienfuegos, A., Geng, J.G., Alvarez-VAllinA, L. \& SANZ, L. (2012). The axonal repellent Slit2 inhibits pericyte migration: Potential implications in angiogenesis. Exp Cell Res 318(4), 371-378.

Hall, M., Gourley, C., Mcneish, I., Ledermann, J., Gore, M., Jayson, G., Perren, T., Rustin, G. \& Kaye, S. (2013). Targeted anti-vascular therapies for ovarian cancer: Current evidence. $\mathrm{Br} J$ Cancer 108(2), 250-258.

Italiano, J.E., Richardson, J.L., Patel-Hett, S., Battinelli, E., Zaslavsky, A., Short, S., Ryeom, S., Folkman, J. \& Klement, G.L. (2008). Angiogenesis is regulated by a novel mechanism: Proand antiangiogenic proteins are organized into separate platelet alpha granules and differentially released. Blood 111(3), 1227-1233. 
Jain, S., Harris, J. \& Ware, J. (2010). Platelets: Linking hemostasis and cancer. Arterioscler Thromb Vasc Biol 30(12), 2362-2367.

Lee, C.C., Liu, K.J. \& Huang, T.S. (2006). Tumor-associated macrophage: Its role in tumor angiogenesis. J Cancer Mol 2(4), 135-140.

Mabjeesh, N.J., Escuin, D., Lavallee, T.M., Pribluda, V.S., Swartz, G.M., Johnson, M.S., Willard, M.T., Zhong, H., Simons, J.W. \& GiannaKakou, P. (2003). 2ME2 inhibits tumor growth and angiogenesis by disrupting microtubules and dysregulating HIF. Cancer Cell 3, 363-375.

Mannello, F. \& Medda, V. (2011). Differential expression of MMP-2 and MMP-9 activity in megakaryocytes and platelets. Blood 118(24), 6470-6471.

Mannello, F. \& Tonti, G.A. (2007). Concise review: No breakthroughs for human mesenchymal and embryonic stem cell culture: Conditioned medium, feeder layer, or feeder-free; medium with fetal calf serum, human serum, or enriched plasma; serum-free, serum replacement nonconditioned medium, or ad hoc formula? All that glitters is not gold!. Stem Cells 25(7), 1603-1609.

Min Soon, C., Bottsford-Miller, J., Vasquez, H.G., Stone, R., Zand, B., Kroll, M.H., SoOd, A.K. \& Afshar-Kharghan, V. (2012). Platelets increase the proliferation of ovarian cancer cells. Blood 120(24), 4869-4872.

Nieswandt, B., Hafner, M., Echtenacher, B. \& Mannel, D.N. (1999). Lysis of tumor cells by natural killer cells in mice is impeded by platelets. Cancer Res 59(6), 1295-1300.

Nkandue, D.S., Mqoco, T.V., Visagie, M.H., Stander, B.A., Wolmarans, E., Cronje, M.J. \& Joubert, A.M. (2013). In vitro changes in mitochondrial potential, aggresome formation and caspase activity by a novel 17-beta-estradiol analogue in breast adenocarcinoma cells. Cell Biochem Funct 31(7), 566-574.

Oida, T. \& Weiner, H.L. (2010). Depletion of TGF- $\beta$ from fetal bovine serum. J Immunol Methods 362(1-2), 195-198.

Pandey, K.B. \& Rizvi, S.I. (2011). Biomarkers of oxidative stress in red blood cells. Biomed Pap Med Fac Univ Palacky Olomouc Czech Repub 155(2), 131-136.

Papetti, M. \& Herman, I.M. (2002). Mechanisms of normal and tumor-derived angiogenesis. Am J Physiol Cell Physiol 282(5), C947-C970.

Peterson, J.E., Zurakowski, D., Italiano, J.E., Michel, L.V., Connors, S., Oenick, M., D'amato, R.J., Klement, G.L. \& FolkMAN, J. (2012). VEGF, PF4 and PDGF are elevated in platelets of colorectal cancer patients. Angiogenesis 15(2), 265-273.

Plake, K.H. \& WARnKe, P.C. (1997). Vascular endothelial growth factor. J Neurooncol 35, 365-372.

Pratheeshkumar, P., Budhraja, A., Son, Y.-O., Wang, X., Zhang, Z., Ding, S., Wang, L., Hitron, A., Lee, J.C., Xu, M., Chen, G., Luo, J. \& SHI, X. (2012). Quercetin inhibits angiogenesis mediated human prostate tumor growth by targeting VEGFR-2 regulated AKT/mTOR/P70S6K signaling pathways. PLoS One 7(10), e47516.

Pretorius, E. (2011). Traditional coating techniques in scanning electron microscopy compared to uncoated charge compensator technology: Looking at human blood fibrin networks with the ZEISS ULTRA Plus FEG-SEM. Microsc Res Tech 74(4), 343-346.

Pretorius, E. (2013). The adaptability of red blood cells. Cardiovasc Diabetol 12(1), 63.
Radziwon-Balicka, A., Medina, C., O’driscoll, L., Treumann, A., Bazou, D., Inkielewicz-Stepniak, I., Radomski, A., Jow, H. \& RADOMSKI, M.W. (2012). Platelets increase survival of adenocarcinoma cells challenged with anticancer drugs: Mechanisms and implications for chemoresistance. $\mathrm{Br} \mathrm{J}$ Pharmacol 167(4), 787-804.

Radziwon-Balicka, A., Moncada De la Rosa, C., Zielnik, B., Doroszko, A. \& Jurasz, P. (2013). Temporal and pharmacological characterization of angiostatin release and generation by human platelets: Implications for endothelial cell migration. PLoS One 8(3), 1-11.

Reikvam, H., Hatfield, K.J., Oyan, A.M., Kalland, K.H., Kittang, A.O. \& Bruserud, O. (2010). Primary human acute myelogenous leukemia cells release matrix metalloproteases and their inhibitors: Release profile and pharmacological modulation. Eur J Haematol 84(3), 239-251.

Repsold, L., Mgoco, T., Wolmarans, E., Nkandeu, S., Theron, J., Piorkowski, T., Du Toit, P., Van Papendorp, D. \& Joubert, A.M. (2014a). Ultrastructural changes of erythrocytes in whole blood after exposure to prospective in silico-designed anticancer agents: A qualitative case study. Biol Res 47(1), 39.

Repsold, L., Pretorius, E. \& Joubert, A.M. (2014b). An estrogen analogue and promising anticancer agent refrains from inducing morphological damage and reactive oxygen species generation in erythrocytes, fibrin and platelets: A pilot study. Cancer Cell Int 14(1), 48.

Sabrkhany, S., Griffioen, A.W. \& Oude Egbrink, M.G. (2011). The role of blood platelets in tumor angiogenesis. Biochim Biophys Acta 1815(2), 189-196.

Seegers, J.C., De Kock, M., Lottering, M.L., Grobler, C.J., Van Papendorp, D.H., Shou, Y., Habbersett, R. \& Lehnert, B.E. (1997). Effects of gamma-linolenic acid and arachidonic acid on cell cycle progression and apoptosis induction in normal and transformed cells. Prostaglandins Leukot Essent Fatty Acids 56(4), 271-280.

Sierko, E. \& Wojtukiewicz, M.Z. (2004). Platelets and angiogenesis in malignancy. Semin Thromb Hemost 30(1), 95-108.

Stander, A., Joubert, F. \& Joubert, A. (2011). Docking, synthesis, and in vitro evaluation of antimitotic estrone analogs. Chem Biol Drug Des 77(3), 173-181.

Stander, B.A., Joubert, F., Tu, C., Sippel, K.H., Mckenna, R. \& Joubert, A.M. (2012). In vitro evaluation of ESE-15-ol, an estradiol analogue with nanomolar antimitotic and carbonic anhydrase inhibitory activity. PLoS One 7(12), e52205.

Stander, B.A., Joubert, F., Tu, C., Sippel, K.H., Mckenna, R. \& Joubert, A.M. (2013). Signaling pathways of ESE-16, an antimitotic and anticarbonic anhydrase estradiol analog, in breast cancer cells. PLoS One 8(1), e53853.

Theron, A.E., Nolte, E.M., Lafanechere, L. \& Joubert, A.M. (2013). Molecular crosstalk between apoptosis and autophagy induced by a novel 2-methoxyestradiol analogue in cervical adenocarcinoma cells. Cancer Cell Int 13, 87.

Trikha, M., Zhou, Z., Timar, J., Raso, E., Kennel, M., Emmell, E. \& NAKADA, M.T. (2002). Multiple roles for platelet GPIIb/IIIa and alphavbeta3 integrins in tumor growth, angiogenesis, and metastasis. Cancer Res 62(10), 2824-2833.

Van Der Valk, J., Brunner, D., De Smet, K., Fex Svenningsen, Å., Honegger, P., Knudsen, L.E., Lindl, T., Noraberg, J., Price, A., Scarino, M.L. \& Gstraunthaler, G. (2010). Optimization of chemically defined cell culture media-Replacing fetal bovine serum in mammalian in vitro methods. Toxicol in Vitro 24(4), 1053-1063. 
Wartiovaara, U., Salven, P., Mikkola, H., Lassila, R., Kaukonen, J., Joukov, V., Orpana, A., Ristimäki, A., Heikinheimo, M., Joensuu, H., Alitalo, K. \& Palotie, A. (1998). Peripheral blood platelets express VEGF-C and VEGF which are released during platelet activation. Thromb Haemost 80(1), 171-175.

Yan, M., Lesyk, G., Radziwon-Balicka, A. \& Jurasz, P. (2014). Pharmacological regulation of platelet factors that influence tumor angiogenesis. Semin Oncol 41(3), 370-377.
Yoon, S.O., PARK, S.J., Yun, C.H. \& ChunG, A.S. (2003). Roles of matrix metalloproteinases in tumor metastasis and angiogenesis. J Biochem Mol Biol 36(1), 128-137.

Yue, T.L., Wang, X., Louden, C.S., Gupta, S., Pillarisetti, K., Gu, J.L., Hart, T.K., Lysko, P.G. \& Feuerstein, G.Z. (1997). 2-Methoxyestradiol, an endogenous estrogen metabolite, induces apoptosis in endothelial cells and inhibits angiogenesis: Possible role for stress-activated protein kinase signaling pathway and Fas expression. Mol Pharmacol 51(6), 951-962. 